PACS 63.20.Pw, 63.20.Ry

\title{
Discrete breathers in crystals: achievements and open problems
}

\author{
S.V. Dmitriev ${ }^{1,2, \dagger}$ \\ †dmitriev.sergey.v@gmail.com \\ ${ }^{1}$ Institute for Metals Superplasticity Problems of RAS, Khalturin St. 39, Ufa 450001, Russia \\ ${ }^{2}$ National Research Tomsk State University, Lenin Avenue, 36, Tomsk 634050, Russia
}

\begin{abstract}
It has long been known that periodic discrete systems containing defects, in addition to traveling waves, allow for the existence of vibrational modes localized on defects. It turned out that if a periodic discrete system is nonlinear, it can support exact solutions in the form of spatially localized vibrational modes even in the absence of defects. Since all the nodes of the system are identical, only a special choice of initial conditions can distinguish the group of nodes, on which such localized mode, called discrete breather (DB), will be excited. Frequency of DB must lie outside the spectrum of small-amplitude traveling waves. Do not resonating with traveling waves and do not losing energy to their excitation, theoretically DB can maintain its vibrational energy forever, in the absence of thermal vibrations and other perturbations. Crystals are nonlinear discrete systems and discovery of DB in them was only a matter of time. Experimental studies of DB run into considerable technical difficulties, and the main tool of their study is by far the atomistic computer simulations. Having gained confidence in the existence of DB in crystals, we still poorly understand their role in solid state physics. This review covers issues specific to the physics of real crystals, which were not considered in the classical works on DB. In particular, it discusses the interaction of moving DB with crystal lattice defects, analyzes the influence of the elastic deformation of the lattice on the DB properties, presents recent works on the effect of nonlinear lattice excitations on the electron subsystem of the crystal, etc.
\end{abstract}

Keywords: discrete breather, crystal lattice, nonlinear dynamics

\section{Introduction}

Three decades ago, an important discovery was made in the physics of nonlinear phenomena. It was shown that the non-linear system of identical discrete particles periodically arranged in the space can support spatially localized vibrational modes [1-3]. The forerunners of this discovery can be called the works on the localization of vibrational energy in molecules and molecular crystals at high excitation levels [4,5], as well as the localization of energy in a nonlinear chain, considered from a continuum point of view [6]. It turned out that the discreteness and nonlinearity of media are the two main ingredients necessary for the existence of spatially localized modes, called discrete breathers (DB) or intrinsic localized modes (ILM). The dimension of the system and the particular form of the interaction potential between the particles, as a rule, affect only properties of $\mathrm{DB}$, but not the very possibility of their existence. Pioneering works on $\mathrm{DB}$ were focused on the strict proof of their existence and stability and most often they were limited to the consideration of one-dimensional chains of particles interacting with the nearest neighbors by simple nonlinear potentials [1-3,7-12].

Mathematical discovery of $\mathrm{DB}$ has raised the question about the existence of such objects in the real world, and after about a decade the reports have started to appear on their experimental observation in nonlinear discrete systems of different physical nature, such as arrays of optical fibers [13-15], atomic wave packets [16], arrays of superconducting Josephson junctions [17-19], periodic nonlinear electrical circuits [20,21], arrays of mechanical cantilever and nanoelectromechanical shuttles [22-26], and others [27,28].

Attention of researchers was also attracted by crystals, because they also belong to discrete nonlinear systems. However, the microscopic size of DB in crystal lattices makes them very difficult for direct experimental observation. Nevertheless, some successful experiments have been carried out, confirming the existence of DB in crystals [29-38].

For specialists in solid state physics and materials science a new research direction has appeared. It is necessary to thoroughly examine the properties of DB in various crystals, their excitation mechanisms, the possibility of their motion along crystal lattice, describe how DB interact with each other, with the crystal lattice defects, with thermal vibrations, and with external fields. On the basis of this knowledge the role of DB in the physics and mechanics of crystals will be understood, and in the future $\mathrm{DB}$ can become a part of the new technologies.

In this review the latest advances in the study of $\mathrm{DB}$ in crystals will be described and open problems will be listed.

\section{Examples of DB in crystals}

Here we present examples of DB in a variety of defectfree crystals, based on the results of molecular dynamics simulations. In the absence of thermal lattice vibrations such localized vibrational modes have a very long lifetime, measured in thousands or more oscillation periods.

Historically, the first is the result for $\mathrm{NaI}$ alkali halide crystal with an ionic type of atomic bonds [39-41]. The 
vibrations with a large amplitude makes one $\mathrm{Na}$ atom in a high-symmetry crystallographic direction $\langle 001\rangle,\langle 011\rangle$ or $\langle 111\rangle$ with a frequency lying in the gap of the phonon spectrum of the crystal, so this DB is called gap DB. DB frequency decreases with increasing amplitude, reflecting a soft type of the nonlinearity of the vibrational mode. The gap in the phonon spectrum exists due to the significant difference of the masses of the crystal components (iodine atom is 5.5 times heavier than the sodium atom).

The following example is DB in covalent crystals Si and Ge, successfully excited in [42] using the Tersoff interatomic potential. This DB has frequency above the gapless phonon spectrum. DB frequency first increases and after reaching the maximum value decreases with increasing amplitude.

It is easy to excite gap DB in ordered alloys with a large difference in the atomic mass of the components, ensuring the existence of a wide gap in the phonon spectrum, e.g., in $\mathrm{Pt}_{3} \mathrm{Al}$ [43-51]. These works were based on the Morse pair interatomic potentials [52]. Note that in $\mathrm{Pt}_{3} \mathrm{Al}$ it is possible to excite both gap $\mathrm{DB}$ and $\mathrm{DB}$ with frequency above the phonon spectrum $[49,50]$. Gap DB is localized predominantly on one atom of aluminum and is immobile. In contrast, the DB with frequency above the phonon spectrum is localized on four to five atoms of aluminum belonging to a close-packed row and can move along the close-packed row. In [49], collisions of mobile DB with each other and with immobile gap DB were studied. The study of DB in the presence of point defects in the $\mathrm{A}_{3} \mathrm{~B}$ crystal structure has been addressed by the authors of [51].

Japanese researchers studied the DB in graphene and carbon nanotubes [53-57]. To excite DB in graphene, a sophisticated procedure was used [53]. DB has frequency above the gapless phonon spectrum and is proved to be unsustainable. The gap in the phonon spectrum of graphene can be opened by homogeneous elastic deformation, allowing for the existence of gap DB [58]. Clusters of gap DB in graphene were studied in [59] where the possibility of energy exchange between the DB in clusters was shown. DB can also exist on the edge of a stretched graphene nanoribbons of the armchair orientation, as shown in [60,61]. DB frequency lies is in the gap of the phonon spectrum, resulting from the application of tensile strain.

$\mathrm{DB}$ in two-dimensional graphane crystal (fully hydrogenated graphene) was analyzed in $[62,63] . \mathrm{DB}$ is formed by one hydrogen atom, oscillating with a large amplitude perpendicular to the graphane sheet. DB frequency lies in the gap of the phonon spectrum and decreases with increasing amplitude [63]. The energy exchange between closely located DB and the effect of temperature on DB lifetime were studied in [64], where it was concluded that DB can participate in the process of graphane dehydrogenation at elevated temperatures. Review on $\mathrm{DB}$ in carbon and hydrocarbon materials was given in [65].

Beginning with the work [66], where for the first time the mobile DB has been successfully excited in fcc Ni and bcc Nb, DB have been actively studied in pure metals [67-71]. DB in all pure metals have the same structure. The atoms located in a close-packed row, oscillate in anti-phase with the nearest neighbors at a frequency above the phonon spectrum. The frequency increases with increasing amplitude of DB.

\section{Molecular dynamics studies on DB}

Let us describe some specific problems in the physics of real crystals, which have not been well studied in the classical pioneering works on $\mathrm{DB}$.

\subsection{Lattice dimension}

One important issue is whether or not the lower boundary of DB energy exists. It was found that for a broad class of Hamiltonian lattices of dimension one, two or three DB can have arbitrarily small energy [72,73].

In the one-dimensional crystals only two types of $\mathrm{DB}$ polarization are possible - along and across the direction of translation. For crystals of higher dimensionality the number of possible directions of vibration of the atoms in the DB increases.

For DB moving in one-dimensional crystals there exists only one direction of motion, while in the two-dimensional and three-dimensional crystals the question of possible directions of motion of DB is an open problem.

\subsection{Long-range interactions}

In many theoretical works on DB only the interaction between neighboring particles was considered [27,28]. However, in crystals interatomic forces can be long-range (e.g., the Coulomb interaction in ionic crystals or metallic chemical bonds). Polynomial Fermi-Pasta-Ulam potential and Toda potential are not suitable to describe the long-range forces in real crystals, because they do not vanish at long distances. Realistic interatomic potentials are hard at small interatomic distances and become soft with increasing distance, reflecting the structure of atoms consisting of nuclei and electron shells.

\subsection{Interaction of $D B$ with lattice defects}

According to the classical definition, DB is a spatially localized vibrational mode in defect-free nonlinear lattice. However, in condensed matter physics and materials science lattice defects play a crucial role. DB can interact directly with the defects that create distortion of the crystal lattice and local changes of density and bond stiffness, as well as indirectly through the excitation of vibrational modes localized on a defect, or by obtaining energy from such modes.

The first studies of the DB interaction with the vacancy were carried out in the framework of a one-dimensional Frenkel-Kontorova model with nonlinear interaction between particles [74,75]. It has been shown that a moving DB can cause vacancy migration. Moreover, a significant role in this process is played by a nonlinear vibrational mode, localized on the atom adjacent to the vacancy [75]. In frame of the same model the interaction of mobile DB with the interstitial atom has been investigated [76]. Scattering of DB on the interstitial atoms leads to its migration, and, in accordance with the experimental results, the interstitial atom is more mobile than the vacancy. Thus, it has been found that DB can increase the mobility of the point defects, which in turn is related to the diffusion (mass transport) and can facilitate dislocation climb. 
The 2D Morse crystal supports moving DB [77], which allows to study their interaction with defects, for example, with vacancy [78]. According to the obtained results it was concluded that DB in the 2D Morse crystal does not cause vacancy migration, but it lowers the energy barrier for migration for about $10^{2}$ atomic vibrations, while the DB-vacancy interaction lasts [78]. DB interaction with vacancies, dislocations and the surface was investigated by molecular dynamics for bcc iron [79]. In all cases it was found that DB interacting with the defect excites a group of atoms near the defect, which may contribute to structural transformations in the crystal.

\subsection{DB at crystal surface}

Atomically flat surface of three-dimensional crystal can be regarded as a two-dimensional periodic system that can support localized nonlinear oscillations, i.e., DB. The steps on the vicinal surface of the three-dimensional crystal have a quasi-one-dimensional periodic structure, which can also support DB. Furthermore, DB may exist on the edge of twodimensional crystals, for example, on graphene nanoribbon edge $[60,61]$. Such DB can contribute to the physics of the surface.

\subsection{Effect of elastic strain of crystal lattice on $D B$}

Uniform elastic deformation of the crystal is able to significantly affect its properties by changing the symmetry of the crystal lattice and the interatomic interactions [80]. Most effectively this method to control the properties of crystals can be applied to nanomaterials (graphene, metallic nanofibers, etc. [57,80-82]) which are capable of withstanding considerable elastic deformation without structural transformation and fracture. The elastic deformation of graphene and graphene nanoribbons induces a gap in the phonon spectrum, which enables the existence of gap DB in these materials [58-61]. The elastic deformation of the order of 0.01 produces a very large effect on the phonon spectra and frequency of gap $\mathrm{DB}$ in the crystal with $\mathrm{NaCl}$ structure [83].

\subsection{Moving $D B$ and their interaction}

Moving DB in a two-dimensional model of crystals with a local potential have been studied in [84-86]. It is known that $\mathrm{DB}$ in pure metals can move at $0.1-0.5$ of the speeds of sound $[66,68,87]$. Highly localized DB in deformed graphene can demonstrate energy exchange [59]. Thus, DB can participate in energy transfer through the crystal. Colliding DB produce even higher spatial localization of energy [88]. Colliding DB can exchange by their energy and momentum $[88,89]$.

\subsection{First-principles simulations}

Molecular dynamics is based on empirical interatomic potentials and the obtained results should be checked with the use of more accurate methods such as quantum-mechanical density functional theory, which takes into account the quantum nature of the electron subsystem of the crystal. The study of DB in graphane crystal has clearly demonstrated the importance of the first-principle calculations $[62,63]$. Recently Lobzenko et al. have conducted the first-principles simulations of the gap DB in a uniformly deformed graphene [90].

\section{Open problems}

In order to outline the scope of possible further research, we list the following poorly studied questions.

1. Development of methods of DB excitation in molecular dynamics simulations. There are several approaches to finding the initial conditions that generate long-lived localized vibrations, for example, the rotating wave approximation, the method of gradient descent, the spontaneous excitation of chaotic DB as a result of modulation instability of certain vibrational modes, by quenching the thermalized lattice, by imposing bell-shaped functions on certain short-wavelength phonon modes in the nonlinear regime. The complexity of this problem is that the same crystal can support DB of various types.

2. Determination of the basic properties of $\mathrm{DB}$ in various crystals at zero temperature: their minimum and maximum energy, frequency range, the degree of spatial localization, the ability or inability to move through the crystal, elastic stress fields created by DB and other physical characteristics. Molecular dynamics can be the main tool for these studies.

3. First principles calculations of DB properties in crystals are very important to clarify the data obtained by the method of molecular dynamics.

4. Evaluation of the probability of spontaneous excitation of DB by thermal fluctuations. Estimation of the concentration and lifetime of DB in thermal equilibrium. Development of numerical methods for identification of DB in thermalized lattices in molecular dynamics calculations.

5. Since the DB are non-linear vibrational modes, their appearance is more probable under intensive external stimulation (radiation, high-gradient temperature field, high electric current density, plastic deformation, phase transitions, etc.), when the crystal receives substantial portions of energy. It is natural to assume that the role of $\mathrm{DB}$ significantly increases in the far from thermodynamic equilibrium processes. Thus, it is important to examine the contribution of DB to the physics of crystals far from equilibrium and under intense external stimulation.

6. Evaluation of the contribution of $\mathrm{DB}$ to the thermal conductivity, electric charge transfer, to the magnetic properties, specific heat, thermal expansion, and other physical properties of crystals.

7. Estimation of the role of DB in the generation of defects and their interaction with the crystal lattice defects.

8. Particularly important is to continue the experimental studies on DB in crystals. The most direct experimental methods analyze the spectra of different waves interacting with the crystals. With the increasing knowledge about the impact of DB on the physical properties of crystals, the new possibilities can be open by measuring the DB-sensitive properties of crystals.

Solving these problems will bring us closer to understanding the role of DB in solid state physics. 


\section{References}

1. A.S. Dolgov. Sov. Phys. Solid State 28, 907 (1986).

2. A.J. Sievers, S. Takeno. Phys. Rev. Lett. 61, 970 (1988).

3. J.B. Page. Phys. Rev. B 41, 7835 (1990).

4. A.A. Ovchinnikov. JETP 57, 263 (1969).

5. A.A. Ovchinnikov, N.S. Erihman. Usp. Fiz. Nauk 138 (2), 289 (1982).

6. A. M. Kosevich, A. S. Kovalev. JETP 40, 891 (1974).

7. R.S. MacKay, S. Aubry. Nonlinearity 7, 1623 (1994).

8. D. Bambusi, Nonlinearity 9, 433 (1996).

9. R. Livi, M. Spicci, R.S. MacKay. Nonlinearity 10, 1421 (1997).

10. G.L. Alfimov, V.A. Brazhnyi, V.V. Konotop. Physica D 194, 127 (2004).

11. K. Yoshimura. Proc. of the Int. Symp. on Nonlinear Theory and its Applications (NOLTA-2015) P. 902 (2015).

12. K. Yoshimura. AIP Conf. Proc. 1474, 59 (2012).

13. H.S. Eisenberg et al. Phys. Rev. Lett. 81, 3383 (1998).

14. D.N. Christodoulides, N.K. Efremidis. Opt. Lett. 27, 568 (2002).

15. J.W. Fleischer et al. Nature (London) 422, 147 (2003).

16. B. Eiermann et al. Phys. Rev. Lett. 92, 230401 (2004).

17. E. Trias, J.J. Mazo, T.P. Orlando. Phys. Rev. Lett. 84, 741 (2000).

18. P. Binder et al. Phys. Rev. Lett. 84, 745 (2000).

19. J.J. Mazo, T.P. Orlando. Chaos 13, 733 (2003).

20. F. Palmero et al. Phys. Rev. E 84, 026605 (2011).

21. L.Q. English et al. Phys. Rev. Lett. 108, 084101 (2012).

22. M. Sato et al. Phys. Rev. Lett. 90, 044102 (2003).

23. J. Wiersig, S. Flach, K.H. Ahn. Appl. Phys. Lett. 93, 222110 (2008).

24. M. Sato et al. Phys. Rev. E 87, 012920 (2013).

25. M. Sato et al. Phys. Rev. Lett. 107, 234101 (2011).

26. M. Sato et al. Chaos 25, 013103 (2015).

27. S. Flach, C.R. Willis. Phys. Rep. 295, 181 (1998).

28. S. Flach, A.V. Gorbach. Phys. Rep. 467, 1 (2008).

29. B.I. Swanson et al. Phys. Rev. Lett. 82, 3288 (1999).

30. N.K. Voulgarakis et al. Phys. Rev. B 64, 020301 (2001).

31. G. Kalosakas, A.R. Bishop, A.P. Shreve. Phys. Rev. B 66, 094303 (2002).

32. D.K. Campbell, S. Flach, Y.S. Kivshar. Phys. Today 57, 43 (2004).

33. M.E. Manley et al. Phys. Rev. B 77, 214305 (2008).

34. M.E. Manley et al. Phys. Rev. Lett. 96, 125501 (2006).

35. M.E. Manley et al. Phys. Rev. B 79, 134304 (2009).

36. M. Kempa et al. J. Phys.: Condens. Matter. 25, 055403 (2013).

37. A.J. Sievers et al. Phys. Rev. B 88, 104305 (2013).

38. J.F.R. Archilla et al. Physica D 297, 56 (2015).

39. S.A. Kiselev, A.J. Sievers. Phys. Rev. B 55, 5755 (1997).

40. L.Z. Khadeeva, S.V. Dmitriev. Phys. Rev. B 81, 214306 (2010).

41. A.A. Kistanov, Y.A. Baimova, S.V. Dmitriev. Tech. Phys. Lett. 38, 676 (2012).

42. N.K. Voulgarakis et al. Phys. Rev. B 69, 113201 (2004).

43. N.N. Medvedev et al. Letters on Materials 3 (1), 34 (2013).

44. P.V. Zakharov et al. Fundamentalnie Problemi Sovremennogo Materialovedeniya 11 (2), 260 (2014).

45. N.N. Medvedev, M.D. Starostenkov, M.E. Manley. J. Appl. Phys. 114, 213506 (2013).

46. N.N. Medvedev et al. Russ. Phys. J. 57 (3), 387 (2014).
47. S.V. Dmitriev et al. Phys. Solid State 52 (7), 1398 (2010).

48. P.V. Zakharov et al. Fundamentalnie Problemi Sovremennogo Materialovedeniya 11 (4), 533 (2014).

49. P.V. Zakharov et al. JETP 148 (2), 252 (2015).

50. N.N.Medvedev et al. Technical Physics Lett. 41, 50 (2015).

51. P.V. Zakharov et al. Fundamentalnie Problemi Sovremennogo Materialovedeniya 12 (2), 146 (2015).

52. A.I. Tsaregorogsev et al. Phys. Met. Metallogr. 58 (2), 336 (1994).

53. Y. Yamayose et al. Europhys. Lett. 80, 40008 (2007).

54. T. Shimada, D. Shirasaki, T. Kitamura. Phys. Rev. B 81, 035401 (2010).

55. T. Shimada et al. Physica D 239, 407 (2010).

56. Y. Kinoshita et al. Phys. Rev. B 77, 024307 (2008).

57. Y. Doi, A. Nakatani. Journal of Solid Mechanics and Materials Engineering 6, 71 (2012).

58. L.Z. Khadeeva, S.V. Dmitriev, Yu.S. Kivshar. JETP Lett. 94, 539 (2011).

59. J.A. Baimova, S.V. Dmitriev, K. Zhou. Europhys. Lett. 100, 36005 (2012).

60. E.A. Korznikova, J.A. Baimova, S.V. Dmitriev. Europhys. Lett. 102, 60004 (2012).

61. E.A. Korznikova et al. JETP Lett. 99, 222 (2012).

62. B. Liu et al. J. Phys. D: Appl. Phys. 46, 305302 (2013).

63. G.M. Chechin et al. Phys. Rev. B 90, 045432 (2014).

64. Yu.A. Baimova et al. JETP 149 (4), 1 (2016).

65. J.A. Baimova et al. Rev. Adv. Mater. Sci. 42, 68 (2015).

66. M. Haas et al. Phys. Rev. B 84, 144303 (2011).

67. V. Hizhnyakov et al. Phys. Scripta 89, 044003 (2014).

68. R.T. Murzaev et al. Comput. Mater. Sci. 98, 88 (2015).

69. A.S. Semenov et al. Fundamentalnie Problemi Sovremennogo Materialovedeniya 12 (1), 26 (2015).

70. S.V. Dmitriev, A.P. Chetverikov, M.G. Velarde. Phys. Stat. Solidi B 252, 1682 (2015).

71. V. Hizhnyakov et al. Nucl. Instrum. Meth. B 303, 91 (2013).

72. M. Kastner. Phys. Rev. Lett. 92, 104301 (2004).

73. M. Kastner. Nonlinearity 17, 1923 (2004).

74. J. Cuevas et al. Phys. Lett. A 315, 364 (2003).

75. J. Cuevas et al. Physica D 216, 115 (2006).

76. J. Cuevas et al. Discrete Contin. Dyn. S. Series S 4 (5), 1057 (2011).

77. A.A. Kistanov et al. JETP Lett. 99 (5-6), 403 (2014).

78. A.A. Kistanov et al. Tech. Phys. Lett. 40 (8), 657 (2014).

79. D.A. Terentyev et al. Modelling Simul. Mater. Sci. Eng. 23, 085007 (2015).

80. T. Zhu, J. Li. Prog. Mater. Sci. 55, 710 (2010).

81. S. Ogata et al. Phys. Rev. B 70, 104104 (2004).

82. J.A. Baimova et al. Phys. Rev. B 86, 035427 (2012).

83. S.V. Dmitriev, J.A. Baimova JTPhys. 81, 71 (2011).

84. J.L. Marin, J.C. Eilbeck, F.M. Russell. Phys. Lett. A 248, 225 (1998).

85. J.L. Marin, F.M. Russell, J.C. Eilbeck. Phys. Lett. A 281, 21 (2001).

86. J. Bajars, J.C. Eilbeck, B. Leimkuhler. Physica D 301-302, 8 (2015).

87. V. Hizhnyakov et al. Springer Series in Materials Science 221, 229 (2015).

88. A.A. Kistanov et al. Eur. Phys. J. B 87, 211 (2014).

89. Y. Doi. Phys. Rev. E 68, 066608 (2003).

90. I.P. Lobzenko et al. Phys. Solid State 58 (3), 616 (2016). 